\title{
LE GENRE DU POUVOIR MÉDIATIQUE
}

\author{
Julie Sedel
}

Editions du Croquant | « Savoir/Agir »

$2018 / 4 \mathrm{~N}^{\circ} 46$ | pages 43 à 50

ISSN 1958-7856

ISBN 9782365121842

Article disponible en ligne à l'adresse :

https://www.cairn.inforevue-savoir-agir-2018-4-page-43.htm

Distribution électronique Cairn.info pour Editions du Croquant.

(C) Editions du Croquant. Tous droits réservés pour tous pays.

La reproduction ou représentation de cet article, notamment par photocopie, n'est autorisée que dans les limites des conditions générales d'utilisation du site ou, le cas échéant, des conditions générales de la licence souscrite par votre établissement. Toute autre reproduction ou représentation, en tout ou partie, sous quelque forme et de quelque manière que ce soit, est interdite sauf accord préalable et écrit de l'éditeur, en dehors des cas prévus par la législation en vigueur en France. Il est précisé que son stockage dans une base de données est également interdit. 


\section{Le genre du pouvoir médiatique}

et article propose de lever le voile sur l'exclusion des femmes du champ des états-majors de médias ${ }^{\mathrm{I}}$. Comment le genre, entendu comme «construction sociale de la différence sexuelle [qui] s'inscrit dans l'économie des rapports sociaux de sexe, structurés par une domination du 'masculin' sur le 'féminin', et évolue dans l'histoire et dans l'espace social $»^{2}$, agit-il au plus haut niveau de la hiérarchie médiatique ? Après avoir montré la façon dont les normes masculines déterminent le poste de dirigeant de média, cet article revient sur les formes de la division sexuelle du travail révélées pas la place des femmes dans les états-majors des médias nationaux. Celle-ci fait apparaître deux types de «ségrégation ", l'une, verticale, la seconde, horizontale. L'article montre ensuite la façon dont le genre agit sur l'organisation du temps et les formes

I. Cet article est tiré de mon Habilitation à diriger des recherches, «Sociologie d'une position patronale : dirigeant de médias », université de Strasbourg, décembre 2018. Ce travail croise des entretiens auprès de dirigeants de médias, une analyse statistique à partir d'un échantillon de 93 dirigeants en poste en 2016, des recherches dans différents fonds d'archives.

2. Anne Revillard, Laure De Verdalle,

«Dynamiques du genre. (introduction)",

Terrains E travaux, vol. 10, $\mathrm{n}^{\circ} 1,2006$.

\author{
JULie Sedel \\ Université de Strasbourg, \\ SAGE, UMR 7363
}

de commandement. Occuper une position dans un secteur où les normes organisationnelles et professionnelles dominantes sont «masculines » et la conserver, nécessite, in fine, de «travailler sa place ${ }^{3} »$.

\section{Un discours androcentré}

Dans les discours journalistiques et académiques, les états-majors des médias nationaux se présentent comme des lieux «neutres », c'est-à-dire indifférents aux rapports sociaux de sexes. Cette invisibilité est renforcée par le caractère androcentré (gender blind $\left.{ }^{4}\right)$ des recherches menées sur les hiérarchies médiatiques. Les femmes sont quasiment absentes des ouvrages consacrés aux dirigeants de médias. Dans une compilation de portraits

3. Marlène Benquet, Jacqueline Laufer, «Femmes dirigeantes », Travail, genre et sociétés vol. 1, $\mathrm{n}^{\circ} 35,2016$, p. 19-25.

4. Érik Neveu, «Le genre du journalisme. Des ambivalences de la féminisation d'une profession ", Politix, vol. 13, n ${ }^{\circ} 51,2000$, p. 177 212. 
de 40 personnalités qui «ont fait la presse » seule une dirigeante figure au palmarès: Marguerite Durand, fondatrice de La Fronde, en 1897, journal féministes. De façon symptomatique, si Françoise Giroud est mentionnée comme formant avec Jean-Jacques Servan-Shreiber, de L'Express «un superbe tandem ", elle reste un personnage de second rang $^{6}$. Par ailleurs, aucun des dix "éditocrates " (la catégorie rassemblant au-delà du périmètre des dirigeants de médias et des journalistes) identifiés par Mona Chollet et al. n'est une femme'. À l'exception de Françoise Giroud, ancienne directrice de $L^{\prime} E x p r e s s^{8}$, les rares figures de dirigeantes de médias auxquelles ont été consacrés un ouvrage sont incarnées par des journalistes présentatrices de télévision?.

5. Sandrine Lévêque, «Femmes, féministes et journalistes: genre et engagement comme ressources professionnelles: le cas des rédactrices de La Fronde » in Béatrice Damian-Gaillard et al. (dirs.), Le journalisme au féminin. Assignations, inventions et stratégies, Rennes, PUR, 2010, p. 47-68.

6. Yves Agnes, «Jean-Jacques Servan Shreiber », in Yves Agnes, Patrick Eveno (dir.), Ils ont fait la presse. L'bistoire des journaux en France en 40 portraits, Paris, Vuibert, 2010.

7. Mona Chollet et al. Les éditocrates. Ou comment parler de (presque) tout en racontant (vraiment) n'importe quoi, Paris, La Découverte, 2009. En revanche, quatre femmes figurent parmi Les nouveaux chiens de garde de Serge Halimi (Paris, Raisons d'Agir, 1997): Michèle Cotta, Anne Sinclair, Claire Chazal et Christine Ockrent.

8. Christine Ockrent, Françoise Giroud, Une ambition française, Paris, Fayard, 2003 ; Laure Adler, Françoise, Paris, Grasset, 2011.

9. Alain Hertoghe, Marc Tronchot, Anne Sinclair, femme de tête, dame de Cour, Paris, Calmann-Lévy, 2011. Trois autres ouvrages ont été consacrés au couple Anne Sinclair et Dominique Strauss-Khan après l'affaire dite du Sofitel.

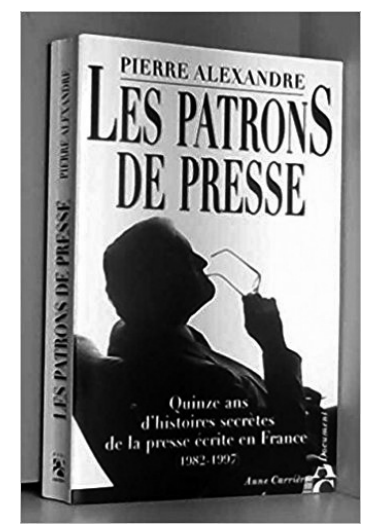

L'iconographie des dirigeants de médias vient appuyer cette représentation genrée du poste. Elle insiste sur le corps au travail, pour bien spécifier la dimension professionnelle, première, de l'identité sociale des dirigeants. Ces mises en scène du pouvoir font ressortir l'ethos masculin paré des attributs de la fonction (costume, cravate, fauteuil, lunettes), comme l'illustre la couverture de l'ouvrage de Pierre Alexandre, Les Patrons de presse ${ }^{\mathrm{I}}$.

"Dame de fer», "Femme de pouvoir ", "dame de pique », "battante », les expressions utilisées par la presse féminine et généraliste pour désigner les dirigeantes de médias montrent la façon dont l'ethos masculin domine la construction d'un crédit. Pour faire leur place, les premières femmes dirigeantes ont été amenées à endosser les signes de la masculinité : cheveux courts, veste tailleurs, pantalon, à être « one of the boys », pour accéder à l'espace public et gagner en légitimité.

Io. Pierre Alexandre, Les Patrons de presse: quinze ans d'histoires secrètes de la presse écrite en France (1982-1997), Paris, Anne Carrière, 1997. 


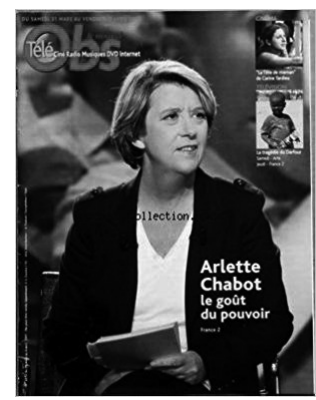

De façon générique, les qualités prêtées par la presse aux dirigeantes de médias renvoient à des oppositions mythico-rituelles: haut / bas, dessus / dessous, sec / humide, chaud / froid, actif / passif, mobile / immobile, qui mettent en évidence les formes de classification sexuées avec lesquelles nous construisons le monde. Le surinvestissement dans le travail, la capacité à "prendre des coups", à se «battre», le «sangfroid », le « culte de l'effort », le goût du "risque ", la franchise, voire la "brutalité », sont autant de traits saillants qui ressortent des portraits et entretiens réalisés auprès des dirigeantes de médias. Ainsi, Delphine Ernotte, première présidente du groupe de télévision publique "sait dire son fait à quelqu'un froidement, droit dans les yeux ", confie, par exemple, son entourage. "Elle peut être dure. Quand elle veut se séparer de quelqu'un, elle sait le faire sans tergiverser ", ajoute cette autre proche, ancienne ministre de la Culture ${ }^{\mathrm{II}}$. La description d'Arlette Chabot, une des rares femmes à avoir dirigé l'information offre un condensé de ces clichés: "La dame de fer de

II. « Delphine Ernotte, la femme qui détient le pouvoir », Marie-Claire, 5 octobre 2016. l'info, qui refuse la langue de bois et use d'un style franc, pour ne pas dire brutal, attire souvent sur elle la foudre. Tous lui reconnaissent un caractère de chien, mais aussi un solide humour et une rigueur sans faille ${ }^{12}$. Le poids de l'ethos viril peut expliquer que l'absence de "féminité » puisse aussi être constituée en ressource comme l'exprime, avec humour, Arlette Chabot: "Quand on n>est pas ravissante à 25 ans, on paraît moins moche à $50 !{ }^{13}$. Ces qualités s'opposent à l'écoute, l'attention, l'émotion, l'empathie, la gentillesse, à l'ordre familial, la reproduction, « au monde des choses humaines " classées comme féminines. "Je persiste à croire qu'une femme est plus capable de suivi, d'attention et d'écoute qu'un homme, dit par exemple, Laure Adler. Les femmes ont une plus grande proximité avec la vie. L'importance des crèches, de l'éducation, de la culture, du social, elles connaissent. ${ }^{14}$ Ainsi, les qualités mises en avant par ces dirigeantes contribuent-elles à perpétuer l'ordre des sexes ou plus exactement à inscrire la division sexuelle dans «l'ordre des choses ${ }^{15}$.

\section{Une ségrégation verticale et horizontale}

Comme dans la majorité des lieux de pouvoirs, la direction de média est

I2. "Arlette Chabot, lutteuse de l'information », Télé Obs, 23 août 2010.

I3. Annick Cojean, «Arlette Chabot, une intervieweuse pure et dure », Le Monde, 24 avril 2007.

I4. «Laure Adler : «À France Culture, j’ai été secrétaire puis directrice ", $J D D, 5$ octobre 2013.

I5. Pierre Bourdieu, La domination masculine, Paris, Seuil, 1998, collection « Liber». 
un poste dessiné pour les hommes. La proportion de femmes au sein de la population des dirigeants de médias (10 femmes sur 93 dirigeants) est équivalente à celle des ambassadrices (18 ambassadrices sur 179), en 2001, et se situe entre le ratio femmes/hommes du Conseil d'Etat (une femme sur sept conseillers), des préfets ( 9 préfètes sur 117 postes) et celui de la Cour des comptes (une femme sur quinze) ${ }^{16}$.

Les femmes occupent souvent une position subalterne dans la division sociale verticale du travail. Aux femmes, les tâches d'exécution, aux hommes, le travail d'incarnation. Françoise Giroud utilise la métaphore de la colonisation pour caractériser les rapports de domination entre les sexes. Prenant acte de la « réussite» du duo qu'elle formait avec Jean-Jacques Servan Schreiber, à la direction de l'Express, elle tirait la conclusion suivante: "un homme et une femme peuvent être des États associés et non colonisés l'un par l'autre ${ }^{17}$. Une dirigeante d'une grande station de radio nationale utilise l'expression de «malédiction » pour désigner cette séparation des rôles assignant aux hommes l'incarnation du média, aux femmes, l'exécution du travail.

Aussi perçoit-elle son poste comme un moyen de se libérer de l'emprise des hommes sur son travail :

«La vraie question c'est: “qu'est-ce

I6. Anicet Le Pors, Françoise Milewski, « Piloter l'accès des femmes aux emplois supérieurs : premier Rapport du Comité de pilotage pour l'égal accès des femmes et des hommes aux emplois supérieurs des fonctions publiques ", Sciences Po publications, 2002, p. 13 cité par Damian-Gaillard et al., Journalismes au féminin, op. cit., p. 267.

I7. Françoise Giroud, Histoire d'une femme libre, Paris, Gallimard, 2013, p. 215. que c'est d'être une femme dirigée par des hommes ?’ D’une certain façon, une fois qu'on est à la direction, on n'a plus ça quoi. Et ça fait...C'est une question qui s'est posée à vous? - Ben oui, parce qu'il y avait quand même l'idée que les mecs sont numéros 1 et les femmes, parce qu'elles ont la tête bien faite, font le travail derrière eux. Donc, d'une certaine façon, moi je me suis délivrée de cette malédiction mais qui était assez répandue » (entretien, 2017).

Les dirigeantes doivent aussi affronter un ensemble de situations, de remarques qui sont autant de rappels à l'ordre des sexes : une rémunération d'un tiers plus faible que leurs homologues masculins, être désignée par son prénom plutôt que par son nom lors de réunions de direction, jugée dénuée de compétences techniques, sous l'influence d'un mentor et plus généralement, peu autonomes dans la conduite des affaires, ce que l'on retrouve également dans le fait, pour les femmes politiques, d'être constamment ramenées à la condition de $«$ mineure $»^{18}$.

L'examen des lieux d'exercice et des types de fonctions exercées par les femmes dirigeantes attestent également d'une ségrégation horizontale. De tous les supports, la presse écrite apparaît comme un bastion de la masculinitér ${ }^{19}$ : seulement $9 \%$ des femmes ont travaillé exclusivement en presse écrite contre $30 \%$ dans l'audiovisuel et $7 \%$

I8. Frédérique Matonti, Le genre présidentiel. Enquête sur l'ordre des sexes en politique, Paris, la Découverte, 2017, p. 34.

I9. Julie Sedel, «Une reconfiguration des spécialisations professionnelles au Monde ", in Damian-Gaillard B. et al., (dirs.), Le journalisme au féminin, op. cit.. 
dans les deux supports (presse écrite et audiovisuelle). La très faible présence des femmes à la direction de médias écrits et parmi les directeurs éditoriaux atteste du caractère fortement masculin de cette fraction du patronat de médias. Les dirigeantes exercent surtout des fonctions de gestion et d'administration (41\% des femmes dirigeantes de médias ne sont pas journalistes contre $23 \%$ des hommes) $)^{20}$.

La féminisation toute relative des états-majors de médias s'explique par le mouvement de nomination des femmes dans l'audiovisuel public (sept sur treize de notre échantillon y travaille) ${ }^{2 \mathrm{I}}$. Sans cet effet collatéral de la mise en place des politiques de parité, la direction de médias resterait une affaire d'hommes.

\section{Une temporalité et un commandement masculins}

Le caractère masculin de la position de dirigeant de médias apparaît de façon saillante à travers la temporalité et le type de commandement. La temporalité est un domaine privilégié où le « genre » comme rapport social agit. L'on retrouve dans les horaires contrai-

20. Dans l'audiovisuel, en 1988, les femmes étaient représentées à la production, aux ressources humaines ou la publicité plutôt que la politique générale des chaînes (Monique Dagnaud, Dominique Mehl, Patrons de chaîne, Paris, CNET, 1990, p. 132).

2I. Delphine Ernotte (France Télévisions), Marie-Eve Malouines ( $L C P)$, Dana Hastier (France 3), Sandrine Treiner (France Culture), Véronique Cayla (Arte), Marie-Christine Saragosse (France TV Monde), Laurence Bloch (France Inter). Les autres dirigeantes de mon échantillon travaillent à Télérama (Emmanuelle Delapierre, Fabienne Pascaud), Lagardère active (Claire Léost), Causeur (Elisabeth Levy), TF1 (Catherine Nayl) et Huffington (Anne Sinclair). gnants de la presse quotidienne, en particulier, certains traits de "l'institution totale $»^{22}$, selon laquelle l'organisation du temps conduit à une perte d'autonomie.

Nathalie Lapeyre a montré comment l'arrivée des femmes dans des professions masculines les confrontait à « un ethos professionnel fondé sur un contrat de genre (macro) spécifique, caractérisé par le surinvestissement des hommes dans l'espace professionnel au détriment de la sphère privée $»^{23}$. Dans un travail antérieur sur la féminisation $\mathrm{du}$ quotidien Le Monde, nous avons souligné que les femmes qui avaient un rapport plus souple au temps étaient aussi les plus dominées au sein de la rédaction ${ }^{24}$. Aussi, l'ancrage sur le lieu de travail apparaissait-il au cours de cette enquête comme un élément de différenciation entre hommes et femmes, ce temps étant nécessaire pour se faire une place, occuper l'espace, glaner des informations utiles pour se construire des positions d'autorité. L'occupation des positions les plus hautes reposait sur l'élasticité du temps de travail que les hommes et quelques femmes s'octroient.

Cet investissement dans le travail se fait souvent au détriment d'autres temps, comme le temps familial, domaine historiquement construit comme féminin : "Je n’ai pas dîné avec mes enfants avant qu'ils aient dix ans »,

22. Erving Goffmann, Asiles. Etude sur les conditions sociales de malades mentaux, Paris, Minuit, 1968 [1961].

23. Nathalie Lapeyre, Les professions face anx enjeux de la féminisation, Toulouse, Octares, 2003, p. 52-53.

24. Julie Sedel, «Une reconfiguration des spécialisations professionnelles au Monde ", art. cit. 
dit ainsi cette dirigeante journalistique politique qui associe sa nouvelle fonction à un rapport moins contraint au temps que lorsqu'elle dirigeait le service politique d'une grande radio nationale. La maternité a longtemps constitué un des principaux obstacles à l'accès aux postes de direction, comme l'exprime Arlette Chabot: "Quand j'étais jeune, s'éloigner quelques mois d'une rédaction pour faire un bébé signifiait qu'on tirait un trait sur sa carrière. C'était “Marche ou crève” ! Résultat, je ne suis pas mariée et je n’ai pas d'enfants... Heureusement, ça ne se passe plus comme ça aujourd'hui $»^{25}$. Un dirigeant décrit le congé maternité comme un « handicap de carrière réel ». L'arrivée d'un enfant impose non seulement des «contraintes horaires » mais aussi « un changement d'aspirations », souligne-t-il :

«Les femmes qui reviennent de congé maternité n'ont plus les mêmes aspirations. Elles veulent retrouver leur poste mais elles ne veulent pas retrouver leur poste. Elles se disent : "attendez, moi maintenant, j'ai la garderie, j’ai ça”. Et évidemment, l'entreprise doit prendre ça en compte » (entretien, 2017).

Tout se passe comme si au dévouement marital et familial s'opposait le dévouement au travail comme l'illustre l'exemple d'une journaliste d'un service international, célibataire dont la vie professionnelle avait « tout dévoré » selon les propos d'un dirigeant (entretien, 2017). Aussi l'investissement professionnel repose-t-il pour une partie de ces dirigeants sur une division sociale

25. "Une journée avec Arlette Chabot », Elle, $1^{\text {er }}$ avril 2011. du travail qui assigne au conjoint ou à l'auxiliaire, le rôle de caring.

L'exercice de l'autorité, la tonalité des relations, dans l'institution renvoient également à des formes de classements genrés. Les états-majors des institutions médiatiques sont des « univers» de commandement. Les dirigeants ne sont pas toujours également préparés aux fonctions de donneurs d'ordre, comme l'exprime cette dirigeante qui «comprend » que les «femmes n'aient pas «envie de mettre l'armure et de castagner ».

Le service militaire, par exemple, met à l'épreuve de la hiérarchie et de l'obéissance, les impétrants, excluant les femmes de ce champ de connaissance par corps. Une dirigeante souligne la façon dont durcir les traits de caractères et adopter une «affectation exagérée de l'autorité » peuvent construire des positions d'autorité dans cet univers masculin. Mais l'adoption d'une forme de «mimétisme radical» rencontre également des limites pour les femmes qui s'y adonnent, l'original étant toujours préféré à la copie ${ }^{26}$.

De façon générique, occuper une position hiérarchique impose de mettre à distance les stigmates associés à la féminité renvoyant au monde de la reproduction, au corps, à l'idée de nature.

\section{Faire sa place}

Devenir dirigeante de média suppose que des conditions objectives et subjectives soient réunies. Les condi-

26. «Elle ne sait pas faire confiance, elle n'écoute pas. Dans les couloirs, on parle d'une dérive poutinienne du pouvoir » relate Alxandre Debouté, «Fronde contre la direction au Monde », Le Figaro, 6 mai 2014. 
tions sociales de possibilité renvoient à la trajectoire familiale, scolaire, professionnelle, amicale, amoureuse, intellectuelle. Parallèlement, l'autoréflexivité, la distance à soi et aux autres, permettent de s'armer face aux préjugés et de se projeter dans un futur réservé aux hommes. Les dirigeantes s'appuient sur des schèmes d'interprétation et des modèles d'identification pour construire leur position. Laure Adler, ancienne dirigeante de France Culture, a fait sa thèse sur les journalistes féministes au XIX ${ }^{e}$ siècle sous la direction de Michèle Perrot, historienne des femmes. Sandrine Treiner, qui dirige la même station de radio, décrit Michèle Perrot comme l'une de «ses professeurs fétiches» à l'université de Jussieu. S'identifier à des figures de dirigeantes, réfléchir à sa position aident à tenir sa place. Les biographies de Françoise Giroud participent ainsi du travail de légitimation du pouvoir au féminin. Françoise Giroud a joué le rôle de mentor pour une génération de femmes dirigeantes (Christine Ockrent, Michèle Cotta). «Elle est une icône. Elle a vécu l'amour libre, a réussi à être indépendante financièrement, a été patronne de journaux avec, sous son autorité, des hommes. Elle aimait la mode et les idées. Françoise Giroud représente la femme complète. Elle est une intellectuelle et une séductrice", déclare Laure Adler ${ }^{27}$. Au passage, cette dirigeante réintroduit, dans son éloge, l'idée de nature, associée aux femmes à celle de culture, classée du côté masculin. Les biographies de cette ancienne dirigeante contribuent à fabriquer cette figure héroïque. Autodidacte, cette

27. "Laure Adler: "J'ai besoin de vivre dans le risque" ", JDD, 8 juillet 2012. orpheline de père va bénéficier de ses entrées dans la société artistique et intellectuelle parisienne pour rejoindre le journalisme féminin puis le journalisme politique.

Sa trajectoire l'amène à ressentir un sentiment d'étrangeté vis-à-vis de sa classe sociale et de son genre qu'elle vit sur le mode du dédoublement :

«Enfant rejetée par la bourgeoisie dont j'étais issue parce que mon milieu d'origine n'aimait pas les pauvres, sinon pour en user, j'ai âprement cherché à y trouver une place. Enfant d'un rebelle, j'ai toujours fui au moment de m'y intégrer et d'en accepter les lois » écritelle [...] Ainsi ai-je vécu, garçon le jour, fille la nuit, androgyne ombrageux, me conduisant obstinément tant à l'égard des hommes qu'à celui de la société comme le loup de la fable, celui qui envie le chien pour la sécurité que lui donne son maître [...] mais qui, découvrant au cou du chien la trace du collier, reprend, affamé, solitaire mais libre, le chemin de la forêt $»^{28}$.

Mais ce type de parcours d'ascension sociale reste exceptionnel au regard des trajectoires modales des dirigeantes de médias entrées dans les années 1970 dans le secteur. Michèle Cotta, Christine Ockrent et Anne Sinclair ont grandi au sein de la bourgeoisie intellectuelle et politique (leurs pères sont respectivement avocat pénaliste, maire SFIO de Nice, ambassadeur en Belgique, industriel en cosmétiques). Toutes les trois appartiennent au champ politique auquel elles ont consacré un grand nombre d'ouvrages (15 pour

28. Fançoise Giroud, Histoire d'une femme libre, op. cit., p. 28. 
Michelle Cotta et Christine Ockrent, 7 ouvrages pour Anne Sinclair) par leur formation à Sciences Po, leur milieu familial et les alliances qu'elles ont contractées avec des acteurs politiques (de premier ou de second plan). Elles ont toutes été décorées de la Légion d'honneur, indice de leur proximité au pouvoir temporel. Comparés à ces pionnières, les parents des dirigeantes de médias nationaux de la génération suivantes sont moins insérés dans la vie politique : leurs pères sont ingénieurs, universitaire, dirigeant d'entreprise, enseignant, médecins, cadre. Les parcours scolaires sont plus diversifiés: elles ont été au lycée de Plaisir, Versailles, Canne et Paris (école bilingue). Une dirigeante a été formée à Sciences Po puis l'ENA, les autres ont suivi une licence à Paris 3, l'école Centrale, une école de commerce, un DEA d'histoire du temps présent de Sciences Po. Leurs conjoints sont comédien, haut fonctionnaire, journaliste, scénariste, mais pas hommes politiques. Autrement dit, tout se passe comme si cette nouvelle génération de femmes dirigeantes s'était affranchie de la figure tutélaire masculine du conjoint ou du père.

En France, la direction de médias est encore une position réservée aux hommes. La reproduction du caractère masculin de cette position s'exerce à travers le rapport au temps, au commandement et renvoie aux ressorts sociaux d'une construction genrée du «charisme ». Les mécanismes qui fondent la croyance et le charisme s'appuyant sur des valeurs de virilité, les femmes sont plus facilement exposées aux risques de discrédit que leurs homologues masculins. Pour la plupart des dirigeantes, la possession d'un stock de connaissances sur le genre leur permet de travailler leur position et de s'imposer pendant un temps dans cet univers masculin. 